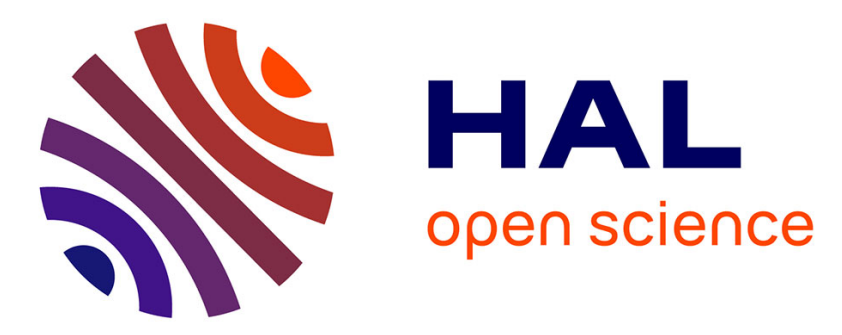

\title{
Localization and imaging of white matter fiber crossings in whole mouse brains using diffusion MRI and serial blockface OCT
}

\author{
Joël Lefebvre, Patrick Delafontaine-Martel, Philippe Lemieux, Maxime \\ Descoteaux, Laurent Petit, Frédéric Lesage
}

\section{To cite this version:}

Joël Lefebvre, Patrick Delafontaine-Martel, Philippe Lemieux, Maxime Descoteaux, Laurent Petit, et al.. Localization and imaging of white matter fiber crossings in whole mouse brains using diffusion MRI and serial blockface OCT. SPIE BiOS,, Mar 2021, On line, United States. 10.1117/12.2577648 . hal-03346656

\section{HAL Id: hal-03346656 \\ https://hal.science/hal-03346656}

Submitted on 16 Sep 2021

HAL is a multi-disciplinary open access archive for the deposit and dissemination of scientific research documents, whether they are published or not. The documents may come from teaching and research institutions in France or abroad, or from public or private research centers.
L'archive ouverte pluridisciplinaire $\mathbf{H A L}$, est destinée au dépôt et à la diffusion de documents scientifiques de niveau recherche, publiés ou non, émanant des établissements d'enseignement et de recherche français ou étrangers, des laboratoires publics ou privés. 


\title{
Localization and imaging of white matter fiber crossings in whole mouse brains using diffusion MRI and serial blockface OCT
}

\author{
Joël Lefebvre*a ${ }^{* a}$ Patrick Delafontaine-Martel ${ }^{\mathrm{b}}$, Philippe Lemieux $^{\mathrm{a}}$, Maxime Descoteaux ${ }^{\mathrm{c}}$, \\ Laurent Petit ${ }^{\mathrm{d}}$, and Frédéric Lesage ${ }^{\mathrm{b}}$ \\ aniversité du Québec à Montréal, Canada \\ ${ }^{b}$ Polytechnique Montréal, Canada \\ ' Université de Sherbrooke, Canada \\ dUniversité de Bordeaux, France
}

\begin{abstract}
To obtain an accurate representation of a brain structural connectivity, diffusion MRI and fiber tracking depend on a good understanding of white matter fiber structures. Although the tracking methods work well when performed in single orientation fiber bundles, most methods are limited in more complex cases, especially to take into account crossing, fanning, and kissing fibers. A recent international fiber tracking challenge concluded that most tracking algorithms generated $4-5$ times more false positive tracks than true tracks on average. This was attributed in large part to a lack of knowledge about the fiber crossing geometry. There is thus a dire need to study more complex fiber geometries to improve the tractography algorithms, for example by classifying those geometries into characteristic crossing topologies (e.g., fanning, curving, bottleneck, pure crossing, ...). Here, we propose a multimodal neuroimaging pipeline to identify and acquire fiber crossing areas in whole mouse brains. Our method uses the Allen Mouse Brain connectivity atlas and tractogram analysis using diffusion MRI techniques to identify candidate regions of interests containing fiber crossings based on two predetermined retrograde viral injection site locations. Based on serial OCT acquisitions, we confirmed the location of crossings. Further experiments will validate in detail the structural nature of crossings using retrograde injections of fluorescent tracers and whole mouse brain serial blockface histology. We believe that this new methodological approach will provide indispensable data for the development of a new generation of tractography algorithms that better resolve complex fiber geometries.
\end{abstract}

Keywords: Whole Brain Imaging, Neurophotonics, Multimodal Imaging, Diffusion MRI, Optical Coherence Tomography, Serial Blockface Histology

\section{INTRODUCTION}

To obtain an accurate representation of the brain structural connectivity, diffusion MRI and fiber tracking depend on a good understanding of white matter fiber structures. Although the tracking methods work well when performed in single orientation fiber bundles, most methods are limited in more complex cases, for example in the presence of crossing, fanning, and kissing fibers. A recent international fiber tracking challenge concluded that across groups, most tracking algorithms could detect $90 \%$ of the true tracks from synthetic brain data, but that all methods consistently generated $4-5$ times more false positive tracks on average. ${ }^{1}$ This was attributed in large part to a lack of knowledge about the fiber crossing geometry. There is thus a dire need to study more complex fiber geometries to improve the tractography algorithms, for example by classifying those geometries into characteristic crossing topologies (e.g., fanning, curving, bottleneck, pure crossing, ... ). Here, we propose a multimodal neuroimaging pipeline to identify and acquire fiber crossing areas in whole mouse brains.

Our imaging pipeline is based on the previously published work. ${ }^{2,3}$ It combines diffusion MRI and dualresolution OCT to acquire whole mouse brains in a fully automated fashion. Here, we focus on the experimental

Further author information: (Send correspondence to lefebvre.joel@uqam.ca) 
preparation phase of this pipeline. Indeed, due to the high amount of data generated by serial histology and to limit the use of animals, one needs to carefully plan these neuroimaging experiments. Our overarching research goal is to study white matter fiber crossing geometry in order to improve the performance of diffusion MRI-based tractography algorithms in those critical areas. To do so, we first need to identify fiber crossing areas in the mouse brain that will be targeted with a high resolution optical microscope sensitive to white matter. One could use diffusion MRI data, obtained before the ex vivo microscopy acquisition, to identify potential fiber crossing areas. Another approach, which we propose in this paper, is to exploit the extensive Allen Mouse brain connectivity atlas. ${ }^{4}$

\section{METHODS}

\subsection{Multimodal imaging pipeline}

The multimodal imaging pipeline is separated into three phases. The initial phase is experiment planning. This consists in using existing datasets and past experiments to identify candidate regions of interest (ROIs) in the mouse brain where we might expect fiber crossings. The second phase of the pipeline, which we presented in previous publications, ${ }^{2,3}$ consists in the actual multimodal acquisition itself. In the previous paper, we used a serial dual-resolution optical coherence tomography (2R-SOCT), combined with diffusion MRI, to image whole mouse brains at high resolution. The third phase consists in data reconstruction and multimodal registration of the OCT and dMRI data. The focus of this conference paper is to present the experimental planning phase that we intend to use to study fiber crossings.

\subsection{Fiber crossing localization}

The Allen Mouse Brain Connectivity Atlas $^{4,5}$ offers data for around 3,000 experiments where a mouse brain was injected with a fluorescent virus which was followed throughout the mouse neurons. This atlas consists in 3D high-resolution map of neural connections in the mouse brain. The axonal projections, originating from various injection sites, were aligned to the Allen Mouse brain common coordinate framework (CCF) to create a neural projection database. This data can be browsed and queried using some of the many modules of the Allen software development kit (SDK). In this paper, we used the neuronal projection paths toward the injection site to identify potential fibers crossing areas. Using the Allen software development kit (SDK), we created a Flask web interface for studying the topology of fibers crossing in mouse brains. This application lets users browse and query experiment data from the Mouse Connectivity Project, as well as looking for potential structures for fiber crossing. The developed platform enables non-programming experts to filter the Allen connectivity Atlas database with a set of search parameters and experimental constraints; this creates an experimental planning map used to assist the microscope operator during the acquisition.

Given two injection site locations our method uses the Allen mouse brain connectivity atlas to identify fiber crossing areas to image. Exploiting retrograde injections from the Allen Mouse brain connectivity dataset, we have selected a list of experiments for which the injection site was in the isocortex of wild-type mice. With this experiment filter, we performed a source search and identified a list of 129 candidate experiments. Then, for each of these experiments we have generated the projection matrix for all the descendant structures of the fiber tracts group in the mouse brain ontological atlas. These amount to 191 fiber structures. They were further separated by hemisphere (left, right, and both), which gives a total of $3 \times 191=573$ structures. Of these, 252 structures did not have any projection signal and were ignored in the analysis. The value within the projection matrix consists in the average fluorescence density within the structure. We defined candidate fiber crossing areas by comparing the projection matrix between multiple retrograde tracer injection experiments. If multiple experiments resulted in high fluorescent expression in the same brain areas, this brain area was selected as a candidate ROIs for imaging.

An additional streamline analysis was performed to better identify the expected crossing geometry (e.g., number of fiber orientations). The streamlines were downloaded from the Allen Institute database and inspected with the MI-Brain software*. The streamline data set was made available through the Neuroinformatics-NL

\footnotetext{
*https://www.imeka.ca/mi-brain/
} 
group $^{\dagger}$. The streamlines were generated from the viral projection maps with a simple marching cube method and they represent the shortest path from any viral projection voxel to the injection site location for each experiment. We performed a Tract Orientation Density Imaging (TODI) analysis ${ }^{6}$ with the viral projection tractograms to obtain fiber orientation distribution function (fODF), which then allowed the use of diffusion MRI methods to evaluate the number of fiber orientations ${ }^{7}(\mathrm{NuFO})$ and of apparent fiber density maximum ${ }^{8}$ (AFD_max).

We combined the information gathered from the Allen connectivity atlas, and from the streamlines analysis and displayed it as a color-coded coronal slice montage (Fig. 1). Simulating an injection experiment, we also displayed two neural projection maps with two different colors, and delineated the expected fiber crossing area with blue overlay. This experimental planning map served as a reference for the microscope operator in the data acquisition phase of the experiment. It was also used to identify 40X OCM mosaics that contained the candidate ROIs.

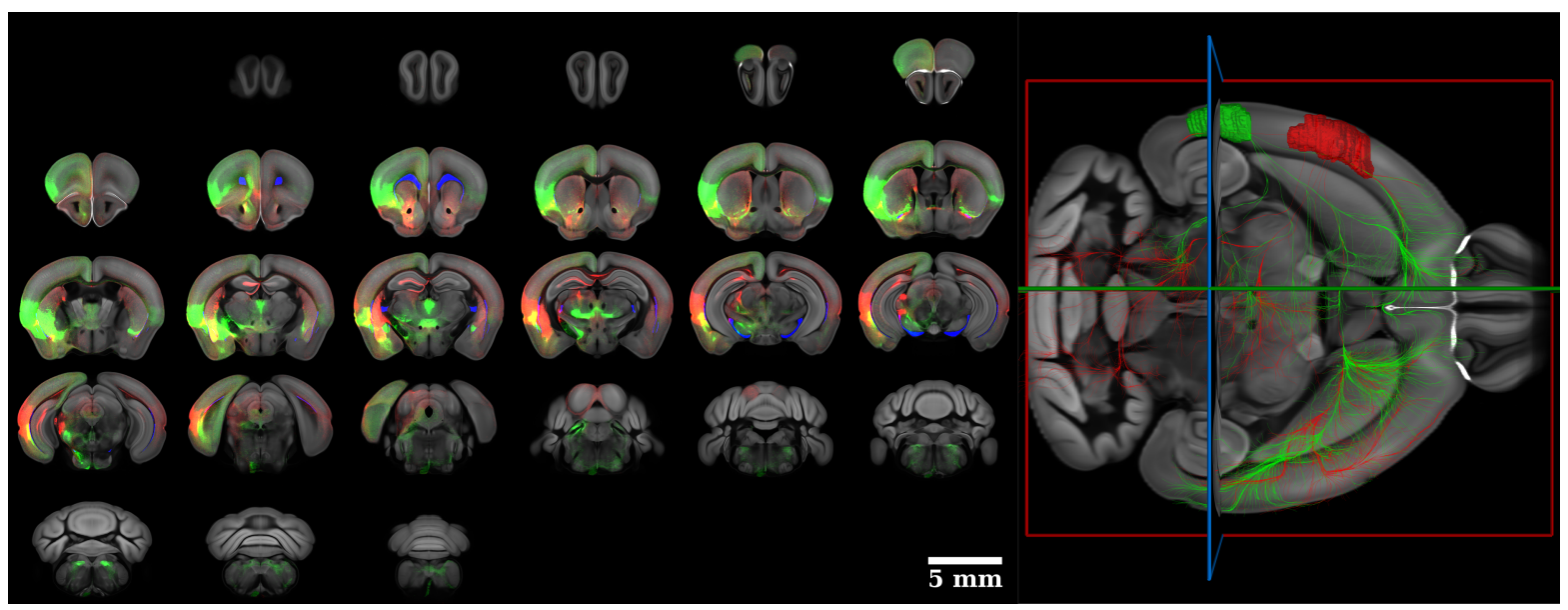

Figure 1. Viral projections (Left) and associated streamlines (Right) for two injection site locations (Red and Green). The data was obtained from the Allen Institute through its AllenSDK python package and was used to target fiber-crossing areas (blue) to image with our system. Here the primary injection structures are the ectorhinal and visceral cortex areas respectively.

\subsection{Data Acquisition}

We used two sources of data to investigate the fiber crossing localization method. First, we exploited 40X OCM mosaics that were acquired with our previously published fully automated dual-resolution serial OCT system. This system consisted in a motorized sample holder coupled with a vibrating blade. The vibratome sequentially removed small 200 microns layers of agarose embedded brain tissue, and then moved the sample under a $3 \mathrm{X}$ objective to acquire a low-resolution mosaic. A coarse image reconstruction was performed on-line and the tissue area was detected using image processing methods. Then the sample was placed automatically under a 40x microscope objective, and 40X optical coherence microscopy (OCM) mosaic acquisitions were performed for this slice. The sequence of vibratome slicing, 3X imaging and 40X imaging was repeated automatically until the whole brain was acquired. With this system, multiple high resolution OCM mosaics were generated in various locations in the mouse brain. Using this procedure, we built a dataset of 345 high resolution mosaics acquired in 6 mouse brains (this data was acquired for another neuroimaging study, and animal ethics protocols were followed). Using this low-res/high-res OCT dataset, we identified 40x OCM mosaics that were acquired within the fiber crossing candidate ROIs identified with the method described in the previous section.

In addition to the 40X OCM mosaics, we also performed new acquisitions in a single mouse brain and targeted four different regions with a $25 \mathrm{x}$ OCM system. ${ }^{9}$ An 8 -month-old mouse's brain has been retrieved after culling

\footnotetext{
${ }^{\dagger}$ https://neuroinformatics.nl/
} 
via transcardiac PFA injection. For fixation in a matrix of agarose (4\%), a custom hook holds the sample steady inside a custom holder used as a mold. The solution of agarose is then heated to 70 degree Celsius until it becomes transparent. The agarose is then poured in the mold to enclose the sample inside the agarose matrix during the cooling. The matrix is then freed from the custom mold and cut with a scalpel at the desired bregma cut. For the imaging, an SD-OCM coupled with a Thorlabs camera via a spectral filter used to locate the area of imaging. To enable 3D imaging, a Newport linear motor stage performs SD-OCM with axial steps of 20 microns, which correspond to the Rayleigh length of the microscope objective (XLPLN-MP 25x, Olympus) at $1310 \mathrm{~nm}$ and a PSF of 4 microns of diameter. The OCM stacks were assembled with the Gabor fusion method. ${ }^{10}$

\section{RESULTS}

As a proof of concept, we selected the two injection experiments that had the highest viral projection density across all fiber tract structures (see Fig. 1). For these, the injections were in the right hemisphere and the four fiber structures exhibiting the largest correlated fluorescence signal, as defined by the product of their respective unionized projection density, were the extreme capsule of the corpus callosum, the amygdala capsule, and both side (left/right hemispheres) of the anterior commissure temporal limb (delineated in blue in Fig. 1).

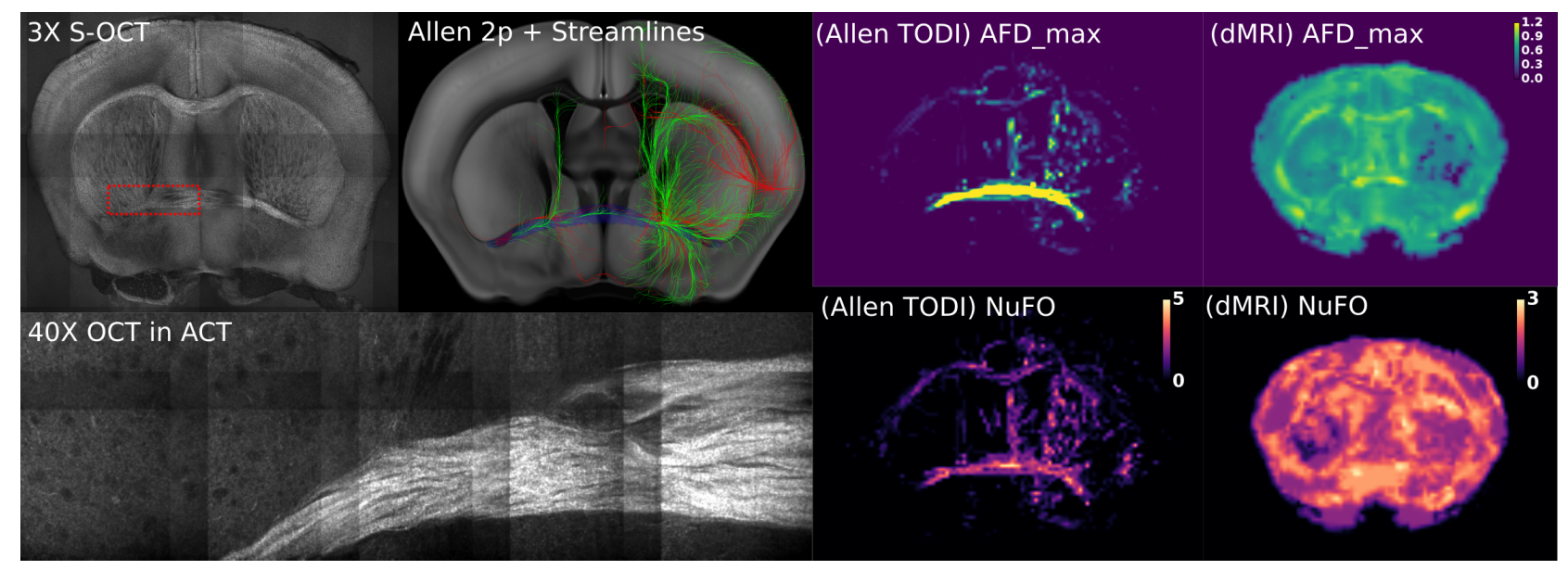

Figure 2. Serial OCT imaging of a whole brain with our previously published dual-resolution OCT system. ${ }^{3}$ High resolution imaging of identified fiber crossing areas reveals complex fiber crossing geometries. Similar observations were made with diffusion MRI acquisitions in the same mouse brain. The Allen connectivity-derived tractogram was analyzed with the TODI method (Allen TODI) of the dMRI scilpy analysis python package ${ }^{\S}$.

Based on this experimental planning map, we then identified 40x OCM mosaics (Fig. 2). Qualitatively we can see that these regions indeed contain complex fiber geometries. We performed a 2D structure tensor analysis to detect the local principal orientations. ${ }^{11}$ To display the orientation, we use the HSV color coding scheme (hue : angle, saturation : tensor energy, value : image intensity). As can be seen in the Fig. 3, the structure tensor was able to detect the main in-plane orientation of the fiber structures.

We also computed local orientation distribution functions (ODFs) using the extracted orientation from the structure tensor analysis (Fig. 3, bottom row). We used neighborhood size of 64 pixels (corresponding to around 200 microns) and we applied an angular smoothing with a Gaussian kernel $(\sigma=3)$. As can be seen, we detect small orientation orientations in tightly packed fiber bundles such as in the anterior commissure. Multiple orientation peaks are observed in areas of fanning geometry.

In addition to automated 40X OCM mosaics, we performed OCT acquisitions in a few regions of mouse brain slice. Those regions were selected by the microscope operator based on the generated experimental planning maps. Those areas are in the corpus callosum, the auditory radiation, and two locations in the endopiriform nucleus (Fig. 4, only the corpus callosum is shown). For the corpus callosum, the assembled OCM volume shows multiple fiber bundles crossings. Upon visual inspection one can see that some fibers overlap others. We are 

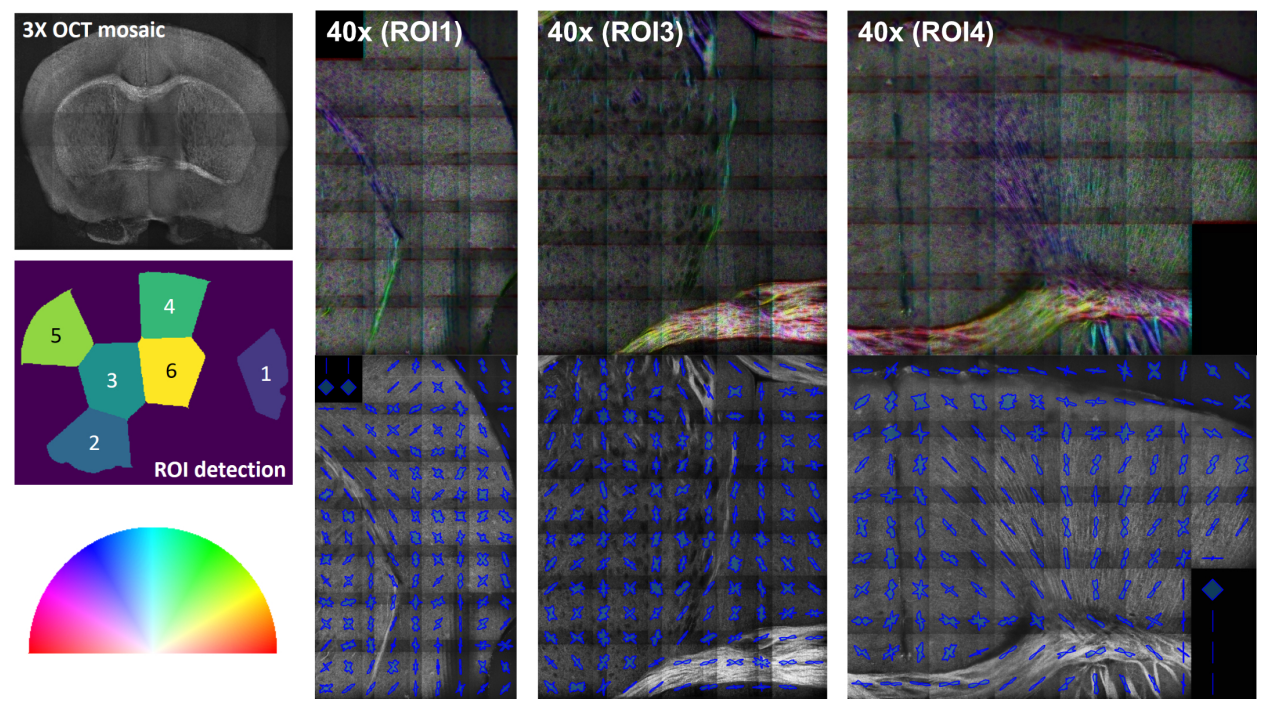

Figure 3. 40X OCM mosaics. (Left) 3X OCT brain slices used to automatically detect 40X ROIs. (Top row) Color coded 40x mosaics (the color wheel indicate the local orientation, as estimated with 2D structure tensor). (Bottom row) Local orientation distribution functions for the corresponding mosaics.

optimistic that this kind of 3D OCM data can be used to distinguish between interleaving, fanning and other crossing geometries. This will be the subject of further investigations.
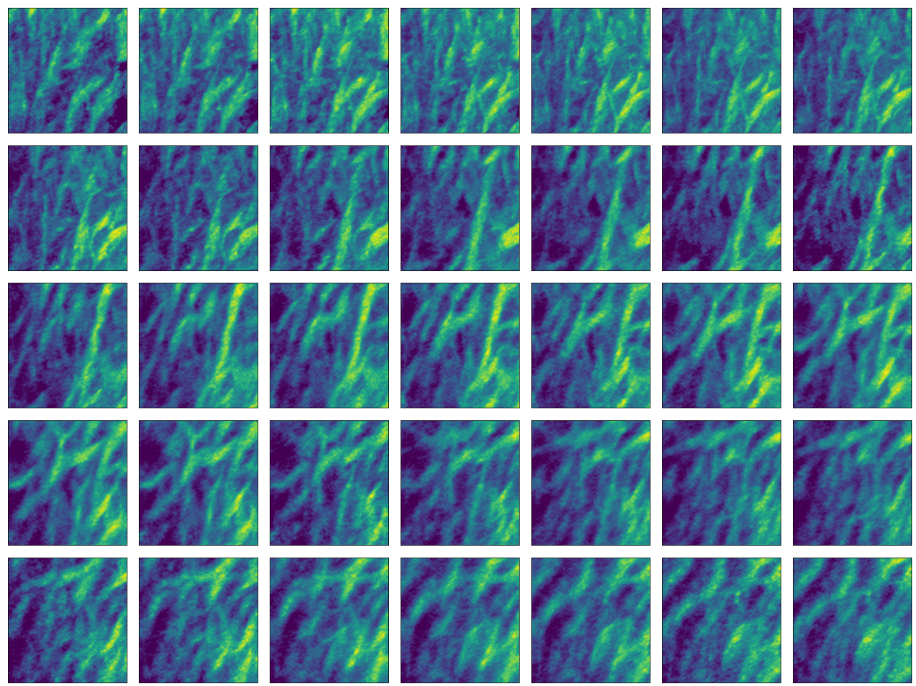

Figure 4. Montage of an assembled 3D OCM acquired in the corpus callosum. Each image represents a different axial depth within the stack, organized in a row-first order. The data was denoised with a non-local mean filter and the intensity was normalized for each slice separately.

\section{DISCUSSION}

In this paper, we made use of an existing public dataset of mouse brain connectivity to identify brain regions that might contain fiber crossings. With this information, we generated experimental planning maps that were used to assist the microscope operator during acquisition. These maps were compared with 40x mosaics that were acquired automatically with a serial OCT system in a previous study. They were also used to guide 3D 
OCM stacks acquisitions in new mouse brain slices. With this data, complex white matter fiber geometries were visualized in $2 \mathrm{D}$ and in 3D. This proposed fiber crossing localization methodology will be part of a multimodal neuroimaging pipeline combining serial OCT with diffusion MRI.

An advantage of our approach is that it reduces the number of animal experiments that are necessary to target specific brain areas by exploiting the extensive database of injection experiments and neural mappings that were performed by the Allen Institute. In addition to a reduction of animal use, which is one of the aims of animal ethics committees, this use of existing datasets could prove beneficial for small research labs and for small-scale animal brain imaging studies.

The experimental planning maps combines regions of interest overlays and expected neural projections for specific injection locations. This map was shown to be useful to assist a microscope operator. This approach was particularly interesting in the context of remote working where precise and reproducible mouse brain imaging experiments need to be communicated to the limited staff present in the imaging lab. In this study, we used these maps to manually target ROIs with OCM in mouse brain slices, and to identify 40x mosaics within an existing serial OCT dataset. To further automatize serial histology, these experimental planning maps could be integrated in the microscope's control interface and combined with computer vision approach to provide in situ information about the ROIs to target. Also these maps could include other information (e.g., expert delineated targets), and other imaging modalities (e.g., diffusion MRI), thus providing other ways of targeting ROIs.

One limitation of our method is that OCT uses intrinsic contrast of brain tissue, as opposed to the 2-photon fluorescence microscopy used to build the Allen connectivity atlas. OCT is more sensitive to myelinated axons and in-plane fiber bundles, but it also has contrast in other regions such as axially inclined fiber bundles, gray matter and other tissues. Instead, 2-photon fluorescence microscopy combined with injections of retrograde tracers can only visualize the projections of a small subset of infected neurons. A consequence is that the fiber crossing areas identified from the Allen connectivity atlas may not be readily visible in OCT. Furthermore, our fiber crossing localization method identified candidate ROIs by verifying if two injection experiments resulted in strong fluorescence co-expression in the same brain areas. This approach detects fiber crossings regardless of its topology (crossing, interleaved, fanning, collinear, ...). To address these limitations, one could use more than 2 injection sites to identify fiber crossings to better emulate the tissue structures visible with OCT, and one could integrate streamlines analysis to better detect and distinguish between expected crossing topologies.

Regarding the 2D structure tensor analysis to extract the principal local orientation, careful interpretation is required. First of all, the filter response greatly depends on its parameters (i.e., pre-smoothing and neighborhood kernel sizes) and on the local size of the structure being analyzed. For example, using a small pre-smoothing size will extract the orientation of small structures and of large structure boundaries, but will result in apparent isotropic orientations inside large fiber bundles. Also, large neighborhood sizes lead to smoother orientation maps at the expense of loss of spatial information. In addition to this parameter sensitivity, we have observed (with synthetic and real data) that isotropic areas were erroneously described by the structure tensor analysis as containing 2 main orientations instead of a uniform distribution of orientations. We believe that this is due to a poor angular resolution of the structure tensor and to a sensitivity bias toward certain orientations. Regardless of the cause of this bias, which would necessitate further investigation, one does need to be careful when using structure tensor derived metrics to identify fiber crossings.

Finally, we performed a TODI analysis ${ }^{6}$ to convert tractograms acquired from the Allen mouse brain connectivity atlas into fiber orientation diffusion functions (fODF). The TODI-derived fODF were then used to estimate the number of fiber orientations $(\mathrm{NuFO})$ and the apparent fiber density maximum (AFD_max). This information was provided with the experimental planning maps. While doing this analysis we observed that certain voxels resulted in enormous fiber density. Upon closer inspection, we realized that the streamlines generated by the 3D marching cube algorithm effectively represent a path within the maximum fluorescent intensity from any point in the neural projection toward the injection site. This represents a bias as all the streamlines will cross the same voxels when performed with neural projections containing single axons and other thin structures. To address this one could perform tractogram cleaning, streamline fusions, or other preprocessing methods to avoid concentrating all streamlines in individual voxels. Integrating more thorough tractogram analysis in the experimental planning map would be an interesting approach to distinguish between various fiber crossing area geometries. 


\section{CONCLUSION}

In this paper we presented a method to identify candidate brain regions containing fiber crossings, which can then be acquired with serial blockface histology or with other imaging modalities. Our method made use of the publicly available Allen mouse brain connectivity atlas to detect brain areas exhibiting fluorescence co-expression between two viral injection experiments. We generated experimental planning maps to assist the microscope operator during the acquisition. Our method was able to target some fiber crossing candidates, even though it lacked discriminability between fiber crossing geometries (such as fanning, interleaving, collinear, etc.). Further work will address this limitation, for example by better analyzing optical modality-derived tractograms and by integrating diffusion MRI data into the experimental planning phase. Future experiments will validate in detail the structural nature of crossings using retrograde injections of fluorescent tracers. We believe that this new methodological approach will provide indispensable data for the development of a new generation of tractography algorithms that better resolve complex fiber geometries. It will empower neuroanatomists and dMRI scientists to ask new questions and to validate the hypotheses used to understand the diffusion signal.

\section{ACKNOWLEDGMENTS}

This work was supported by a Québec Bioimaging Network (QBIN) pilot research project grant (35450) and by a FRQNT team research project grant (2021-PR-282231).

We also wish to express our gratitude to all the open science efforts, such as the mouse brain images and the connectivity data shared by the Allen Mouse Brain Institute and by the Neuroinformatics-NL group. Open science democratizes the access to mouse brain data and enables the pursuit of various neuroimaging experiments

and novel questions. It is particularly useful in this time of uncertainty related to COVID-19, where many labs and research groups have limited access to animals, imaging facilities and other resources.

\section{REFERENCES}

[1] Maier-Hein, K. H., Neher, P. F., Houde, J.-C., Côté, M.-A., Garyfallidis, E., Zhong, J., Chamberland, M., Yeh, F.-C., Lin, Y.-C., Ji, Q., Reddick, W. E., Glass, J. O., Chen, D. Q., Feng, Y., Gao, C., Wu, Y., Ma, J., He, R., Li, Q., Westin, C.-F., Deslauriers-Gauthier, S., González, J. O. O., Paquette, M., St-Jean, S., Girard, G., Rheault, F., Sidhu, J., Tax, C. M. W., Guo, F., Mesri, H. Y., Dávid, S., Froeling, M., Heemskerk, A. M., Leemans, A., Boré, A., Pinsard, B., Bedetti, C., Desrosiers, M., Brambati, S., Doyon, J., Sarica, A., Vasta, R., Cerasa, A., Quattrone, A., Yeatman, J., Khan, A. R., Hodges, W., Alexander, S., Romascano, D., Barakovic, M., Auría, A., Esteban, O., Lemkaddem, A., Thiran, J.-P., Cetingul, H. E., Odry, B. L., Mailhe, B., Nadar, M. S., Pizzagalli, F., Prasad, G., Villalon-Reina, J. E., Galvis, J., Thompson, P. M., Requejo, F. D. S., Laguna, P. L., Lacerda, L. M., Barrett, R., Dell'Acqua, F., Catani, M., Petit, L., Caruyer, E., Daducci, A., Dyrby, T. B., Holland-Letz, T., Hilgetag, C. C., Stieltjes, B., and Descoteaux, M., "The challenge of mapping the human connectome based on diffusion tractography," Nature Communications $\mathbf{8}$, 1349 (Nov. 2017). Number: 1 Publisher: Nature Publishing Group.

[2] Lefebvre, J., Castonguay, A., Pouliot, P., Descoteaux, M., and Lesage, F., "Whole mouse brain imaging using optical coherence tomography: reconstruction, normalization, segmentation, and comparison with diffusion MRI," Neurophotonics 4(4), 41501 (2017).

[3] Lefebvre, J., Delafontaine-Martel, P., Pouliot, P., Girouard, H., Descoteaux, M., and Lesage, F., "Fully automated dual-resolution serial optical coherence tomography aimed at diffusion MRI validation in whole mouse brains," Neurophotonics 5, 045004 (Nov. 2018). Publisher: International Society for Optics and Photonics.

[4] Oh, S. W., Harris, J. A., Ng, L., Winslow, B., Cain, N., Mihalas, S., Wang, Q., Lau, C., Kuan, L., Henry, A. M., Mortrud, M. T., Ouellette, B., Nguyen, T. N., Sorensen, S. A., Slaughterbeck, C. R., Wakeman, W., Li, Y., Feng, D., Ho, A., Nicholas, E., Hirokawa, K. E., Bohn, P., Joines, K. M., Peng, H., Hawrylycz, M. J., Phillips, J. W., Hohmann, J. G., Wohnoutka, P., Gerfen, C. R., Koch, C., Bernard, A., Dang, C., Jones, A. R., and Zeng, H., "A mesoscale connectome of the mouse brain.," Nature 508, 207-14 (apr 2014).

[5] Allen Institute for Brain Science, "Allen Mouse Brain Connectivity Atlas : Technical White Paper : Overview," Tech. Rep. March, Allen Institute, Seattle, WA (2017). 
[6] Dhollander, T., Emsell, L., Van Hecke, W., Maes, F., Sunaert, S., and Suetens, P., "Track orientation density imaging (TODI) and track orientation distribution (TOD) based tractography," NeuroImage $\mathbf{9 4}$, 312-336 (2014).

[7] Dell'Acqua, F., Simmons, A., Williams, S. C. R., and Catani, M., "Can spherical deconvolution provide more information than fiber orientations? Hindrance modulated orientational anisotropy, a true-tract specific index to characterize white matter diffusion," Human Brain Mapping 34(10), 2464-2483 (2013).

[8] Raffelt, D., Tournier, J.-D., Rose, S., Ridgway, G. R., Henderson, R., Crozier, S., Salvado, O., and Connelly, A., "Apparent Fibre Density: A novel measure for the analysis of diffusion-weighted magnetic resonance images," NeuroImage 59, 3976-3994 (feb 2012).

[9] Marchand, P. J., Lu, X., Zhang, C., and Lesage, F., "Validation of red blood cell flux and velocity estimations based on optical coherence tomography intensity fluctuations," Scientific Reports 10, 19584 (Nov. 2020). Number: 1 Publisher: Nature Publishing Group.

[10] Rolland, J. P., Meemon, P., Murali, S., Thompson, K. P., and Lee, K.-s., "Gabor-based fusion technique for Optical Coherence Microscopy," Optics Express 18, 3632 (feb 2010).

[11] Wang, H., Lenglet, C., and Akkin, T., "Structure tensor analysis of serial optical coherence scanner images for mapping fiber orientations and tractography in the brain," Journal of Biomedical Optics 20(3), 036003 (2015). 